\title{
Intraoperative neurophysiological monitoring for intradural extramedullary spinal tumors: predictive value and relevance of $D$-wave amplitude on surgical outcome during a 10-year experience
}

\author{
Reza Ghadirpour, MD, 1 Davide Nasi, MD, ${ }^{1,2}$ Corrado laccarino, MD, ${ }^{1}$ Antonio Romano, MD, ${ }^{1}$ \\ Luisa Motti, MD, ${ }^{3}$ Rossella Sabadini, MD, ${ }^{3}$ Franco Valzania, MD, ${ }^{3}$ and Franco Servadei, MD 4
}

1'Department of Neurosurgery, University Hospital of Parma, and Department of Neurosurgery, Institute for Scientific and Care Research "ASMN" of Reggio Emilia; '2Department of Neurosurgery, Umberto I General Hospital, Università Politecnica delle Marche, Ancona; ${ }^{3}$ Department of Neurophysiology, IRCCS "Arcispedale Santa Maria Nuova" of Reggio Emilia; and "Department of Neurosurgery, Humanitas Research Hospital and University, Milan, Italy

OBJECTIVE The purpose of this study was to evaluate the technical feasibility, accuracy, and relevance on surgical outcome of D-wave monitoring combined with somatosensory evoked potentials (SSEPs) and motor evoked potentials (MEPs) during resection of intradural extramedullary (IDEM) spinal tumors.

METHODS Clinical and intraoperative neurophysiological monitoring (IONM) data obtained in 108 consecutive patients who underwent surgery for IDEM tumors at the Institute for Scientific and Care Research "ASMN" of Reggio Emilia, Italy, were prospectively entered into a database and retrospectively analyzed. The IONM included SSEPs, MEPs, andwhenever possible-D-waves. All patients were evaluated using the modified McCormick Scale at admission and at 3, 6, and 12 months of follow-up .

RESULTS A total of 108 patients were included in this study. A monitorable D-wave was achieved in 71 of the 77 patients harboring cervical and thoracic IDEM tumors (92.2\%). Recording of D-waves in IDEM tumors was significantly associated only with a preoperative deeply compromised neurological status evaluated using the modified McCormick Scale $(p=0.04)$. Overall, significant IONM changes were registered in $14(12.96 \%)$ of 108 patients and 9 of these patients (8.33\%) had permanent loss of at least one of the 3 evoked potentials. In 7 patients $(6.48 \%)$, the presence of an s18278 caudal D-wave was predictive of a favorable long-term motor outcome even when the MEPs and/or SSEPs were lost during IDEM tumor resection. However, in 2 cases (1.85\%) the D-wave permanently decreased by approximately $50 \%$, and surgery was definitively abandoned to prevent permanent paraplegia. Cumulatively, SSEP, MEP, and D-wave monitoring significantly predicted postoperative deficits ( $p=0.0001$; AUC $=0.905$ ), with a sensitivity of $85.7 \%$ and a specificity of $97 \%$. Comparing the area under the receiver operating characteristic curves of these tests, D-waves appeared to have a significantly greater predictive value than MEPs and especially SSEPs alone ( 0.992 vs 0.798 vs 0.653 ; $p=0.023$ and $p<0.001$, respectively). On multiple logistic regression, the independent risk factors associated with significant IONM changes in the entire population were age older than 65 years and an anterolateral location of the tumor $(p<0.0001)$.

CONCLUSIONS D-wave monitoring was feasible in all patients without severe preoperative motor deficits. D-waves demonstrated a statistically significant higher ability to predict postoperative deficits compared with SSEPs and MEPS alone and allowed us to proceed with IDEM tumor resection, even in cases of SSEP and/or MEP loss. Patients older than 65 years and with anterolateral IDEM tumors can benefit most from the use of IONM.

https://thejns.org/doi/abs/10.3171/2018.7.SPINE18278

KEYWORDS somatosensory evoked potentials; SSEP; motor evoked potentials; MEP; D-wave; intradural extramedullary tumor; diagnostic technique

ABBREVIATIONS AUC = area under the ROC curve; IDEM = intradural extramedullary; IONM = intraoperative neurophysiological monitoring; ISCT = intramedullary spinal cord tumor; MEP = motor evoked potential; NPV = negative predictive value; $P P V=$ positive predictive value; $R O C=$ receiver operating characteristic; $S S E P=$ somatosensory evoked potential.

SUBMITTED March 5, 2018. ACCEPTED July 19, 2018.

INCLUDE WHEN CITING Published online November 9, 2018; DOI: 10.3171/2018.7.SPINE18278. 
I NTRADURAL extramedullary (IDEM) tumors represent $30 \%$ of all spinal tumors. ${ }^{10}$ The most common primitive IDEM tumors are meningiomas and nerve sheath tumors (schwannomas and neurofibromas). ${ }^{24}$ IDEM tumors are almost always benign, and the treatment of choice is surgery with the aim of gross-total removal and spinal cord decompression. ${ }^{12}$ Currently, patients affected by this benign tumor are expected to achieve a complete disease cure with a good quality of life. However, a recent review of surgical complications of spinal meningioma reported a rate of permanent neurological deterioration of $6 \%$ (range $0 \%-21 \%$ ) after surgery. ${ }^{25}$ In this scenario, intraoperative neurophysiological monitoring (IONM) may be a valuable tool in detecting a neurological injury in time for corrective measures to be taken before irreversible damage occurs and in predicting postoperative neurological deficits., ${ }^{5,6}$ Multimodality IONM includes somatosensory evoked potentials (SSEPs), motor evoked potentials (MEPs), D-waves, and electromyography.

The high sensitivity and specificity of IONM have been key factors in establishing its central role in maximal resection of intramedullary spinal cord tumors, with minimal compromise of spinal cord function and improved patient outcomes. ${ }^{1-3,7,11,13,14,16,17,23}$ Currently, few papers have addressed IONM monitorability and accuracy in IDEM tumor surgery with complete IONM modalities. The few studies that have assessed the utility of IONM during IDEM tumor surgery either did not report the use of Dwaves or reported D-waves only sporadically in a limited cohort of patients. ${ }^{3,7,9,13,15-17}$

The purposes of this study were to 1) investigate the rate of IONM ability, especially of D-waves, and evaluate the preoperative factors predicting the possibility of Dwave recording; 2) assess the accuracy of IONM (SSEPs, MEPs, and D-waves) in predicting new postoperative neurological deficits after IDEM tumor resection and statistically compare the accuracy of the 3 modalities by using receiver operating characteristic (ROC) curves; and 3) identify the subgroup of patients with IDEM tumors who are at the highest risk of evoked potential changes during surgery based on uni- and multivariate analyses and who can benefit most from the use of IONM.

\section{Methods}

\section{Patient Population}

From March 2007 to March 2016 clinical, surgical, and IONM data obtained in 108 consecutive patients presenting with IDEM and treated in the Neurosurgery Department at the Institute for Scientific and Care Research "ASMN" of Reggio Emilia, Italy, were prospectively collected in a database and retrospectively analyzed.

Baseline characteristics, including sex, age, predominant spinal level of operation, location of the lesion relative to the spinal cord (anterolateral or posterior), histology, neurological status (assessed using the modified McCormick Scale) on admission, preoperative neurophysiological evaluation with SSEPs and MEPs, and surgical approach, were collected and evaluated.

Surgery for mass lesions such as cysts, vascular malformations, and syringes were excluded from the study.
The diagnosis of an IDEM tumor was performed for all patients using 1.5-T MRI with and without contrast. All patients underwent neurological evaluation using the modified McCormick Scale at admission, postoperatively prior to discharge, and at the 3-, 6-, and 12-month clinical follow-ups. Follow-up between 3 and 6 months also included spinal MRI.

\section{Intraoperative Neurophysiological Monitoring}

Our standardized protocol for IONM during surgery has been described in detail in a previous paper ${ }^{7}$ and includes SSEPs, MEPs, and D-waves. Also, we used electromyography and bulbocavernosus reflex for cauda equina or filum terminale procedures; data obtained using these monitoring techniques were not reported because they do not fall within the scope of this study.

At our institution, a dedicated anesthetic protocol for interventions with IONM includes orotracheal intubation without pancuronium and induction of anesthesia with propofol and remifentanil without halogenated anesthetics. Anesthesia is then maintained with propofol (100-150 $\mathrm{g} / \mathrm{kg} / \mathrm{min}$ ) and fentanyl ( $\mathrm{g} / \mathrm{kg} / \mathrm{hr})$ infusions, avoiding bolus doses. Anesthesia depth control is monitored using an entropy sensor. This protocol minimizes the interaction of drugs with IONM and facilitates a rapid awakening with the possibility of early neurological assessment.

\section{Surgical Procedure and Interpretation of IONM Findings}

The IONM alarm criteria used in our institution are based on current literature ${ }^{4,5,18,19}$ and were described in detail in a previous paper. ${ }^{7}$ Briefly, the criteria are as follows: 1) Persistent amplitude loss of at least $50 \%$ of cortical SSEPs is used as a warning criterion. 2) Persistent MEP loss is considered significant, and surgery is temporarily stopped. 3) A decrease of more than $50 \%$ of the baseline amplitude of the caudal D-wave is considered a warning criterion for permanent motor deficit. 4) The combination of MEPs and D-waves is used to predict motor outcomes in the short and long terms. The disappearance of MEPs with preservation of the D-wave amplitude at more than $50 \%$ of the initial value reflects a transient postoperative motor deficit in the affected limb, and tumor resection may continue if total removal is the goal.

\section{Postoperative Deficits and Follow-Up}

All changes in IONM were correlated with postoperative and follow-up neurological status. All new motor deficits were defined as permanent or transitory depending on whether they were present at the 6-month follow-up. All new postoperative motor deficits were the primary outcomes of this study. Finally, all patients were reevaluated at 12 months after surgery.

\section{Statistical Analysis}

Continuous variables were compared using the Student t-test, while discrete variables were compared using the chi-square test. Each IONM modality's accuracy was determined by comparing intraoperative evoked potential changes with the presence of new postoperative motor deficits via ROC curves. Then a pairwise t-test was used 
to compare the ROC curves of the SSEPs, MEPs, and $\mathrm{D}$-waves. Finally, we used a multiple logistic regression to identify independent risk factors associated with IONM changes during surgery in the entire population. All statistical analyses were performed using IBM SPSS (version 21 , IBM Corp.). A priori statistical significance was set at $\mathrm{p}<0.05$.

\section{Results}

A total of 108 patients who underwent IDEM tumor resections with complete IONM were included. Results of baseline characteristics, including sex, age, predominant spinal level of operation, location of the lesion relative to the spinal cord (anterolateral or posterior), histology, modified McCormick Scale grade on admission, preoperative neurophysiological evaluation with SSEPs and MEPs, and surgical approach, are summarized in Table 1.

\section{Monitorability of IONM}

SSEP and MEP monitoring was performed in all patients. Recording of D-waves was achieved in 71 of 77 the patients harboring cervical and thoracic IDEM tumors (92.2\%). In 6 patients (7.8\%) with severe neurological deficits before surgery ( 2 patients with modified McCormick Scale grade III, 3 with grade IV, and 1 with grade V), the D-wave amplitude was not obtained.

At univariate analysis, the recording of D-waves in IDEM tumors was significantly associated only with preoperative neurological status evaluated using the modified McCormick Scale $(\mathrm{p}=0.04)$. The other tested factors, including sex, age, predominant spinal level of operation, location of the lesion relative to the spinal cord (anterolateral or posterior), preoperative SSEPs and MEPs, surgical approach, and tumor histology, were not statistically predictive of $\mathrm{D}$-wave monitorability.

\section{IONM Changes, Surgical Strategy, and Clinical Correlations}

Overall significant IONM changes (at least 1 evoked potential modality) were registered in $14(12.96 \%)$ of the 108 patients. In 5 patients (4.62\%), these events resolved to baseline levels after temporary stop of surgery and grosstotal resection could be achieved (stop-and-go surgery). In the other 9 patients $(8.33 \%)$, we noted permanent loss of at least one of the 3 evoked potentials. However, only in 2 cases $(1.85 \%)$ were the SSEPs and MEPs lost and the D-wave permanently dropped by about $50 \%$. In these 2 cases, after several attempts to restart the surgery with a change in strategy, the operation was definitively abandoned to prevent permanent paraplegia (stop surgery), and a residual tumor was left in place (Fig. 1). These patients, described in our previous study on $\mathrm{IONM}^{7}$, presented with a persistent new motor deficit at the 6-month follow-up. However, these patients recovered their preoperative neurological status at the 12-month follow-up. In the other 7 patients (7.40\%), the D-wave was stable or decreased no more than $50 \%$ in combination with alteration of MEPs and/or SSEPs and allowed us to the continue surgery, because neurophysiological literature ${ }^{5,18,19}$ suggests that patients usually wake up after the opera-
TABLE 1. Baseline characteristics of 108 patients with IDEM tumors

\begin{tabular}{|c|c|}
\hline Characteristic & Value \\
\hline Mean age, yrs (range) & $55.8(17-81)$ \\
\hline \multicolumn{2}{|l|}{ No. of patients } \\
\hline Male & $51(47.22)$ \\
\hline Female & $57(52.78)$ \\
\hline Total & $108(100)$ \\
\hline \multicolumn{2}{|l|}{ Level } \\
\hline Craniocervical junction & $7(6.48)$ \\
\hline Cervical & $20(18.52)$ \\
\hline Thoracic & $50(46.30)$ \\
\hline Lumbar & $31(28.70)$ \\
\hline Total & $108(100)$ \\
\hline \multicolumn{2}{|l|}{ Location } \\
\hline Anterolateral to spinal cord & $32(29.63)$ \\
\hline Posterior to spinal cord & $76(70.37)$ \\
\hline Total & $108(100)$ \\
\hline \multicolumn{2}{|l|}{ Pathology } \\
\hline Schwannoma & $47(43.52)$ \\
\hline Meningioma & $42(38.89)$ \\
\hline Ependymoma cauda/filum & $14(12.96)$ \\
\hline Dermoid cyst & $4(3.70)$ \\
\hline Solitary fibrous tumor & $1(0.93)$ \\
\hline Total & $108(100)$ \\
\hline \multicolumn{2}{|c|}{ Modified McCormick Scale grade at admission } \\
\hline I & $34(31.48)$ \\
\hline II & $40(37.04)$ \\
\hline III & $28(25.93)$ \\
\hline IV & $5(4.63)$ \\
\hline V & $1(0.93)$ \\
\hline Total & $108(100)$ \\
\hline \multicolumn{2}{|l|}{ Preop SSEPs \& MEPs } \\
\hline Normal & $23(21.30)$ \\
\hline Pathological & $85(78.70)$ \\
\hline Total & $108(100)$ \\
\hline \multicolumn{2}{|l|}{ Surgical approach } \\
\hline Laminectomy & $70(64.81)$ \\
\hline Hemilaminectomy & $26(24.07)$ \\
\hline Laminoplasty & $12(11.11)$ \\
\hline Total & $108(100)$ \\
\hline
\end{tabular}

Values are presented as the number of patients (\%) unless stated otherwise.

tion with a motor deficit, but invariably recover over a period of days or weeks (Fig. 2). Among these patients, 4 emerged from surgery with a new transitory motor deficit while the other 3 patients had no postoperative deficit (false positive).

In the remaining 94 patients $(87.04 \%)$, the combined use of SSEP, MEP, and D-wave IONM invariably predicted a good neurological outcome in all but 1 patient. In this last patient, who harbored a T12-L1 meningioma, the 


\section{Cranial Caudal Lower-Limbs Lower-Limbs \\ D-Wave D-Wave \\ MEP \\ SEP}

Vast.L Tib. AL AbdHL Vast.R AbdHR Tib. AR

Baseline

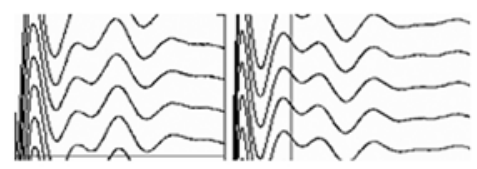

Laminectomy
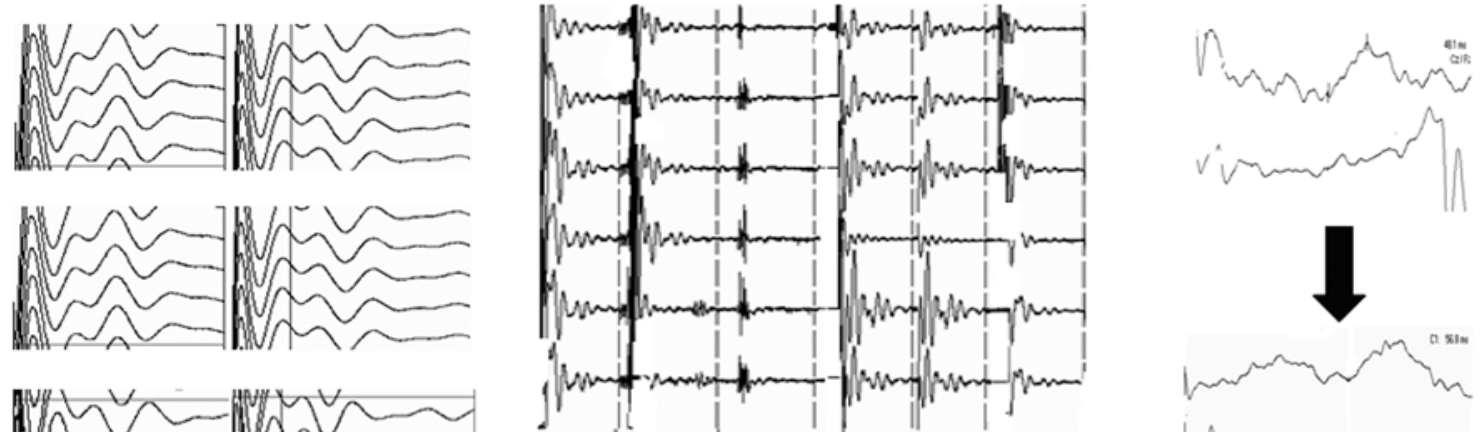

Tumor Removal

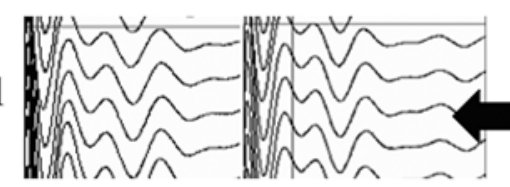

Stop Surgery
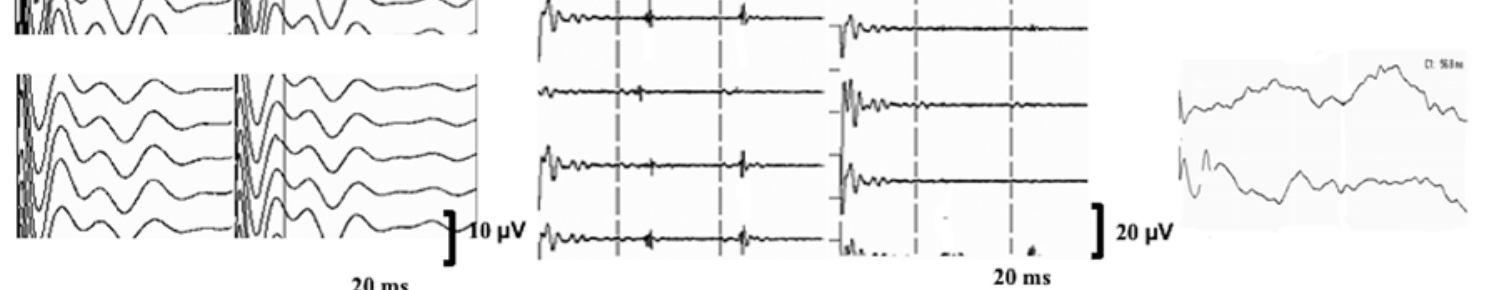

FIG. 1. IONM recordings in a patient harboring a T7-8 solitary fibrous tumor. During tumor removal, the D-wave permanently decreased by about 50\% (black arrow, left) the MEP disappeared (black arrow, center), and the SSEP increased in latency (black arrow, right). In this case, after several attempts to start again and to change surgical strategy, the operation was definitively abandoned to prevent permanent paraplegia (stop surgery), and a residual tumor was left in place. AbdHL = left abductor hallucis nerve; $\mathrm{AbdHR}=$ right abductor hallucis nerve; $\mathrm{SEP}=$ somatosensory evoked potential; Tib. $\mathrm{AL}=$ left anterior tibialis nerve; Tib. $\mathrm{AR}$ $=$ right anterior tibialis nerve; Vast. $L$ = left vastus lateralis; Vast. $R=$ right vastus lateralis.

D-wave could not be monitored, and during surgery the MEP appeared stable. However, after surgery, the patient experienced a transient worsening of paraparesis that recovered completely before discharge (false negative).

The mean preoperative modified McCormick Scale grade improved from 2.14 to 1.34 at 6 months postoperatively. Moreover, we have not found any relationship between the duration of surgery and the presence of significant alterations of IONM or loss of accuracy.

Then we correlated each evoked potential change with neurological outcome.

\section{D-Wave}

Of the 68 patients with a recordable and stable D-wave, none had new permanent motor deficits after surgery at follow-up (no false-negative findings). Indeed, in this group of patients, D-wave monitoring provided relevant data regarding motor outcome and allowed us to proceed with surgery even in cases of MEP/SSEP loss. In 7 $(9.86 \%)$ of 71 patients, the D-wave was stable or decreased no more than $50 \%$ in combination with alteration of MEPs and/or SSEPs and allowed us to continue surgery, because neurophysiological literature predicted no new deficit or transient postoperative motor deficits.

In 2 patients (described above), the $\mathrm{D}$-wave permanently decreased by approximately $50 \%$ with concomitant complete loss of lower-limb SSEPs and MEPs. After several attempts to restart the procedure, surgery was stopped to avoid irreversible and complete motor deficits of the lower limbs. In these cases, D-wave monitoring combined with MEPs and SSEPs allowed us to prevent complete paraplegia at the end of surgery. In another case, the recording of the D-wave was difficult and not stable during the surgical procedure, and at the end of tumor resection the D-wave was diminished by more than $50 \%$, while the MEP and SSEP remained stable. All 3 patients emerged from surgery without any deficit, and we considered the results of registration of D-wave as false positive.

\section{SSEP}

Of 108 patients in whom SSEPs could be monitored, 99 (91.67\%) displayed stable SSEP recordings during surgery. Four $(4.21 \%)$ of the 95 patients presented with a new functional deficit in the immediate postoperative stage (false negative). Two recovered to their preoperative status during hospitalization, while the other 2 patients experienced resolution before the 3-month follow-up.

Of 9 patients with permanent SSEP loss, only 3 displayed a functional deficit (true positive) in the immediate postoperative stage, including 1 patient whose deficits resolved during hospitalization (new transitory deficit) and 2 who presented with deficits at the 6-month follow-up (new permanent deficit). These last 2 patients also had MEP and D-wave permanent loss with subsequent stop of the surgi- 


\section{Cranial D-Wave \\ Caudal D-Wave}

Lower-Limbs

MEP

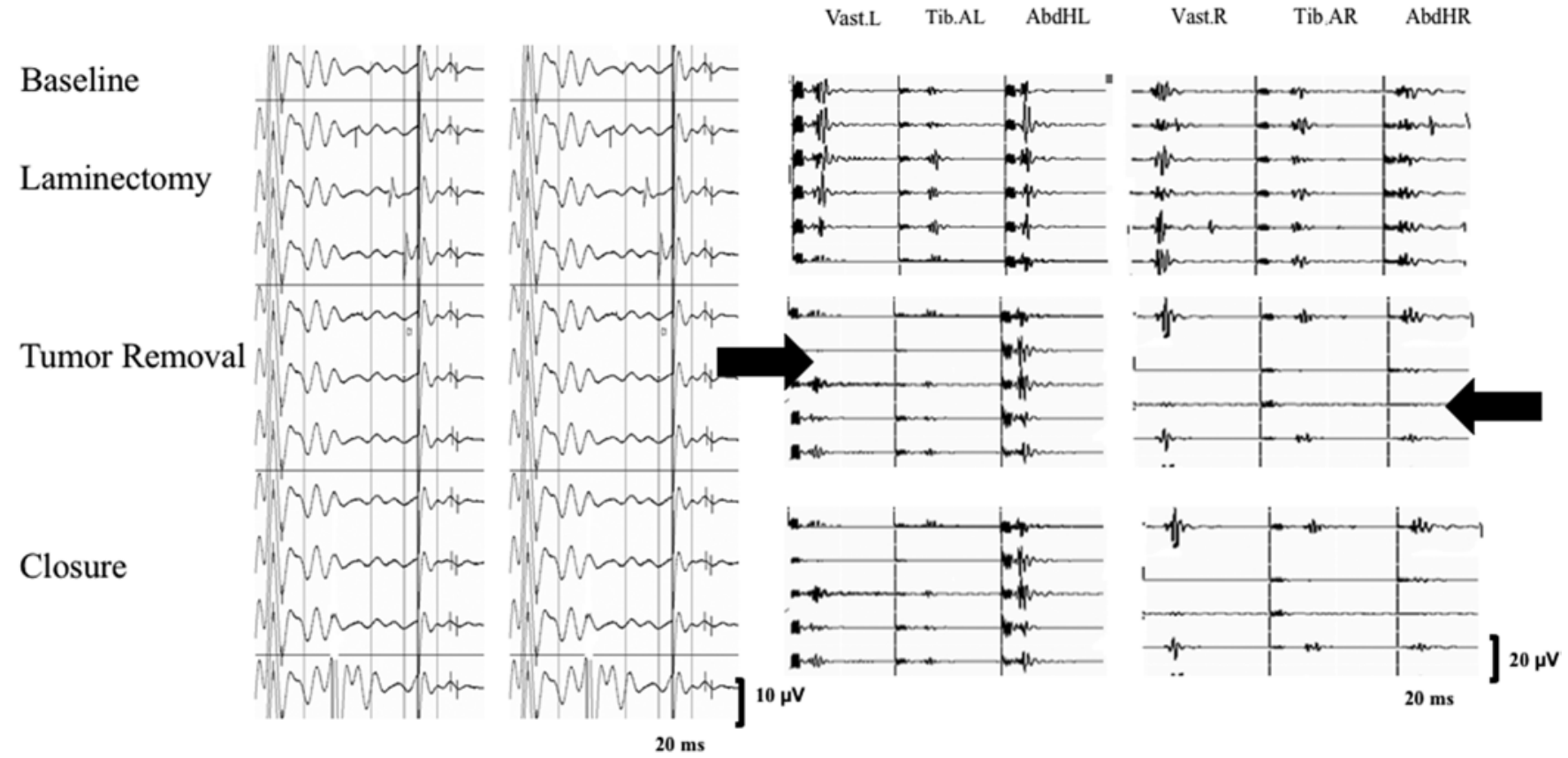

FIG. 2. IONM recording in a patient harboring a T5 anterolateral meningioma. During tumor removal, the MEP disappeared on both sides (black arrows), while the D-wave remained stable. In this patient, the stable D-wave allowed us to continue surgery, because neurophysiological literature suggests that patients usually wake up after the operation with a motor deficit but invariably recover over a period of days or weeks.

cal procedure (described above). The other 6 patients with permanent SSEP alterations during surgery had no postoperative deficit (false positive).

\section{MEP}

Overall, 94 of 97 patients with stable MEP data emerged from surgery neurologically intact (true negative), while 3 patients (3.09\%) experienced deficits in the immediate postoperative stage (false negative), which resolved during hospitalization. Among these patients, in 2 the D-wave was decreased no more than $50 \%$ and, at the end of surgery, the unexpected deficit was foreseen as transitory, while in the other patient, the D-wave was not monitorable and the patient awoke with an unexpected deficit (the case was already illustrated in the previous section).

Of 8 patients with significant MEP changes, 4 had no postoperative deficits (false positive). The other 4 patients emerged from surgery with a new motor deficit: 2 of the deficits resolved during hospitalization and 2 impairments remained at the 6-month follow-up. These 2 patients are those who also had concomitant SSEP and D-wave loss as described above.

\section{Sensitivity, Specificity, and ROC Curves}

Sensitivity, specificity, positive predictive value (PPV), negative predictive value (NPV), positive likelihood ratio, and negative likelihood ratio analyses of each evoked potential are shown in Table 2.
Cumulatively, SSEP, MEP, and D-wave neuromonitoring (multimodal IONM) significantly predicted postoperative deficits $(\mathrm{p}=0.0001)$, with a sensitivity of $85.70 \%$ and a specificity of $97 \%$. However, D-waves demonstrated a significantly higher sensitivity (100\%) than MEPs (62.5\%) and SSEPs (37.5\%). These tests' specificities were $98.40 \%$, $97.03 \%$, and $93.07 \%$, respectively.

Comparing the area under ROC curves (AUCs) of these evoked potentials in 71 patients harboring cervical and thoracic IDEM tumors (where all 3 modalities of IONM were registered) using the pairwise t-test, D-wave monitoring appeared to have higher accuracy and ability to predict postoperative deficits with strong statistical significance compared with MEP and SSEP alone (0.992 vs 0.719 vs $0.523 ; \mathrm{p}=0.023$ and $\mathrm{p}<0.001)$. The ROC curves are displayed in Fig. 3 and these results are summarized in Table 3 .

\section{Factors Correlated With IONM Changes}

At univariate analysis, factors that are significantly associated with a high probability of IONM changes during surgery were age older than 65 years $(\mathrm{p}=0.03)$, anterolateral location to the spinal cord ( $\mathrm{p}<0.0001)$, and meningioma histology $(\mathrm{p}=0.022)$. On multiple logistic regression, the only independent risk factors associated with significant IONM changes in the entire population were the location anterolateral to the spinal cord $(p=0.0001)$ and age older than 65 years $(\mathrm{p}=0.0001)$. These results are summarized in Table 4. 
TABLE 2. Sensitivity, specificity, PPV, NPV, positive and negative likelihood ratios, AUC, and statistical analysis of IONM

\begin{tabular}{|c|c|c|c|c|}
\hline & Multimodal IONM (SSEP + MEP + D-Wave) & SSEP & MEP & D-Wave \\
\hline True negative & 98 & 95 & 97 & 68 \\
\hline True positive & 6 & 3 & 4 & 2 \\
\hline False negative & 1 & 4 & 3 & 0 \\
\hline False positive & 3 & 6 & 4 & 1 \\
\hline Sensitivity & $85.70 \%$ & $37.5 \%$ & $62.5 \%$ & $100 \%$ \\
\hline Specificity & $97 \%$ & $93.07 \%$ & $97.03 \%$ & $98.40 \%$ \\
\hline PPV & $66.67 \%$ & $33.33 \%$ & $66.67 \%$ & $66.67 \%$ \\
\hline NPV & $98.99 \%$ & $95.95 \%$ & $97 \%$ & $100 \%$ \\
\hline LR+ & 28.857 & 5.41 & 21.04 & 64.000 \\
\hline LR- & 0.147 & 0.67 & 0.39 & 0.000 \\
\hline AUC & 0.905 & 0.653 & 0.798 & 0.992 \\
\hline $95 \% \mathrm{Cl}$ & $0.843-0.976$ & $0.556-0.741$ & $0.710-0.869$ & $0.934-0.996$ \\
\hline$p$ value & 0.0001 & 0.16 & 0.002 & 0.0001 \\
\hline
\end{tabular}

$\mathrm{LR+}=$ positive likelihood ratio; $\mathrm{LR}-=$ negative likelihood ratio.

\section{Discussion}

\section{Monitorability of D-Waves}

The rate of the ability to monitor the D-wave has largely been investigated in patients with intramedullary spinal cord tumors (ISCTs). ${ }^{4,19,20}$ Indeed, a critical issue of the use of the D-wave in ISCT is its recording. ${ }^{18}$ In about $30 \%$ of patients harboring ISCTs, the D-wave is not recordable at the beginning of surgery. ${ }^{4,19,20}$ At the same time, muscle MEPs are recordable, even in patients who may not necessarily have a major motor deficit. This phenomenon is well known and described as a de-synchronization of the $\mathrm{D}$-wave, $, 18,19$ and it is due to the fact that fast fibers of the corticospinal tract conduct D-waves with different speeds

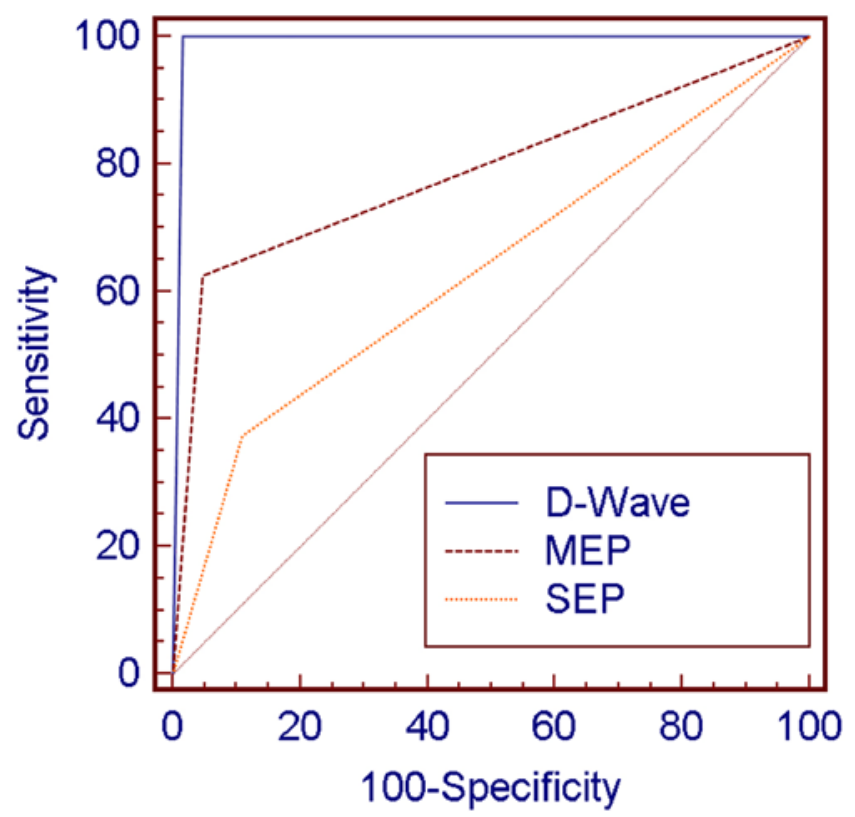

FIG. 3. ROC curves for the D-wave, MEP, and SSEP. Figure is available in color online only. over the lesion site. This commonly happens either after irradiation of the spinal cord or in cases in which ISCTs are associated with extended syringomyelic cysts. ${ }^{4,18,19}$

Sala et al. ${ }^{19}$ in a landmark historical control study about the IONM in ISCT, obtained the recording of the D-wave in about $80 \%$ of patients with a preoperative modified McCormick Scale grade I or II. Conversely, monitorability was poor for patients with severe neurological impairment before surgery (only 3 of 10 patients with McCormick grade III or IV). The authors concluded that there is a clear correlation between preoperative neurological status and monitorability of the D-wave.

To the best of our knowledge, the rate and determinants of D-wave recording in patients with IDEM tumors have

TABLE 3. Comparison between D-Wave, SSEP, and MEP ROC curves in 71 patients harboring cervical and thoracic IDEM tumors

\begin{tabular}{cc}
\hline & Value \\
\hline D-wave vs SSEP & \\
\hline D-wave AUC & 0.992 \\
\hline SSEP AUC & 0.523 \\
\hline Difference btwn AUCs & 0.469 \\
\hline SE & 0.118 \\
\hline 95\% Cl & $0.237-0.701$ \\
\hline p value & $<0.001^{*}$ \\
\hline D-wave vs MEP & \\
\hline D-wave AUC & 0.992 \\
\hline MEP AUC & 0.719 \\
\hline Difference btwn AUCs & 0.273 \\
\hline SE & 0.120 \\
\hline $95 \%$ Cl & $0.038-0.508$ \\
\hline p value & $0.023^{*}$ \\
\hline
\end{tabular}

*Pairwise t-test. 
TABLE 4. Univariate and multivariate analysis of factors associated with a high probability of IONM changes during surgery

\begin{tabular}{|c|c|c|c|c|c|c|c|c|}
\hline & \multirow{2}{*}{$\begin{array}{c}\text { No. of } \\
\text { Patients }\end{array}$} & \multirow{2}{*}{$\begin{array}{c}\text { IOM } \\
\text { Changes }\end{array}$} & \multirow{2}{*}{$\begin{array}{l}\text { No IOM } \\
\text { Changes }\end{array}$} & \multicolumn{3}{|c|}{ Univariate Analysis } & \multicolumn{2}{|c|}{ Multivariate Analysis } \\
\hline & & & & $p$ Value & OR & $\mathrm{Cl} 95 \%$ & $p$ Value & SE \\
\hline Age, yrs & & & & 0.03 & 3.97 & $1.06-14.88$ & 0.0001 & 0.058 \\
\hline$>65$ & 62 & 12 & 50 & & & & & \\
\hline$\leq 65$ & 46 & 2 & 44 & & & & & \\
\hline Total & 108 & 14 & 94 & & & & & \\
\hline Sex & & & & 0.43 & 1.53 & $0.53-4.46$ & 0.453 & 0.065 \\
\hline Male & 51 & 8 & 43 & & & & & \\
\hline Female & 57 & 6 & 51 & & & & & \\
\hline Total & 108 & 14 & 94 & & & & & \\
\hline Level & & & & 0.88 & 0.91 & $0.25-3.28$ & 0.115 & 0.041 \\
\hline Cervical & 27 & 4 & 23 & & & & & \\
\hline Thoracic & 50 & 9 & 41 & & & & & \\
\hline Lumbar & 31 & 1 & 30 & & & & & \\
\hline Total & 108 & 14 & 94 & & & & & \\
\hline Location & & & & $<0.0001$ & 10.8 & $3.14-37.15$ & 0.0001 & 0.083 \\
\hline Anterolateral to spinal cord & 32 & 12 & 20 & & & & & \\
\hline Posterior to spinal cord & 76 & 2 & 74 & & & & & \\
\hline Total & 108 & 14 & 94 & & & & & \\
\hline Pathology & & & & 0.022 & 0.25 & $0.07-0.87$ & 0.21 & 0.045 \\
\hline Schwannoma & 47 & 3 & 44 & & & & & \\
\hline Meningioma & 41 & 10 & 31 & & & & & \\
\hline Other & 20 & 1 & 19 & & & & & \\
\hline Total & 108 & 14 & 94 & & & & & \\
\hline Modified McCormick Scale grade at admission & & & & 0.98 & 1.01 & $0.33-3.10$ & 0.09 & 0.0665 \\
\hline | or II & 74 & 9 & 65 & & & & & \\
\hline III-V & 34 & 5 & 29 & & & & & \\
\hline Total & 108 & 14 & 94 & & & & & \\
\hline
\end{tabular}

Boldface type indicates statistical significance.

not been specifically evaluated until now. $3,7,9,13,15-17$ Only Costa et al., ${ }^{3}$ in their analysis of SSEP, MEP, and D-wave monitoring in 103 spinal cord procedures (23 ISCTs, 55 IDEM tumors, and 25 myelopathies), reported a $97.1 \%$ rate of D-wave monitorability. Indeed, caudal D-wave tracings were obtained in 97 patients and resulted in the most recordable intraoperative neurophysiological parameter in this study, even in compromised patients in whom baseline MEPs were absent. However, the factors predicting D-wave monitorability were not statistically evaluated. In our study, monitorability of the D-wave in IDEM tumors was significantly associated only with preoperative neurological status according to the modified McCormick Scale $(\mathrm{p}=0.04)$. This result confirmed that patients who arrive to surgery with a severe neurological disability had a lower monitorability rate than patients who were neurologically intact. We also know that in these patients with severe neurological deficits, the clinical significance of monitoring aimed to prevent neurological deterioration is limited.

\section{IONM Accuracy in IDEM Tumor Surgery}

Although D-wave monitoring is considered the gold standard for assessing the integrity of the corticospinal tract in spinal monitoring, no study in the literature has specifically addressed the issue of D-wave accuracy in IDEM tumor surgery. ${ }^{21}$

In 2015, Korn et al. ${ }^{13}$ examined IONM results in 100 patients with IDEM tumors. Multimodal IONM demonstrated a high level of accuracy, with sensitivity and specificity as high as $82 \%$ and $95 \%$, respectively, PPV of $82 \%$, and NPV of 95\%. These authors concluded that IONM is feasible and useful in the treatment of IDEM tumors, particularly in identifying injury to the spinal cord. However, the D-wave was successfully monitored only in 18 of 100 patients; thus, the paper does not provide consistent and strong results about the accuracy of the D-wave in IDEM tumors.

In our study, comparing the AUC of each evoked potential, the D-wave appeared to have a greater predictive value than that for MEP and especially SSEP $(p=0.023$ and $\mathrm{p}<0.001$, respectively). On the basis of these results, the recording of the $\mathrm{D}$-wave significantly increased the accuracy of neurophysiological monitoring in IDEM.

\section{Clinical Value of IONM in IDEM Tumor Surgery}

The real clinical value of IONM on the neurological 
outcome after IDEM tumor surgery is unclear and very difficult to prove based on retrospective series or control studies.

In this scenario, the recent Guidelines for the use of electrophysiological monitoring for surgery of the human spinal column and spinal cord published in $2017^{8}$ recommend the use of multimodality intraoperative monitoring, including SSEP and MEP recording, during spinal cord/ spinal column surgery only as a diagnostic adjunct to assess spinal cord integrity. Indeed, these guidelines report that the use of IONM does not result in improved neurological outcomes, even when evoked potential alerts occur.

These recommendations were based essentially on the presence of 2 class II medical evidence studies that refuted the potential therapeutic role of IONM; moreover, the authors considered the other 25 class III medical evidence studies reviewed as inconclusive, inconsistent, and statistically flawed. The first of these 2 studies was published by Choi et al. ${ }^{2}$ and retrospectively reviewed 76 patients who underwent resection of intramedullary spinal cord tumors. Fifty patients underwent tumor resection with IONM, and 26 patients underwent resection without IONM (control group). The authors found no statistically significant difference in postoperative neurological outcome with the use of IONM during intramedullary spinal cord surgery. In the second paper, by Harel et al., ${ }^{9}$ the authors presented a historical control study about the use of IONM without D-wave monitoring in IDEM tumor surgery. They retrospectively compared the neurological outcome after IDEM tumor resection in a group of 41 monitored patients with that of the historical control group of 70 patients who underwent surgery without IONM. In this study, $10 \%$ of patients in the monitored study group developed neurological deficits after surgery compared with $14 \%$ of the historical cohort with no IONM ( $p=0.75)$. On the basis of these results, limited only in the postoperative period, the authors concluded that, while IONM predicts neurological deficits with a high level of accuracy, the same monitoring is not effective in preventing new neurological deficits, and its role in IDEM tumor surgery remains debatable.

In neither study was the use of D-waves reported, which, on the basis of previous literature and the results of our study, is a critical limitation in neuromonitoring during spinal tumor surgery. Indeed, in our series, no patients with monitorable $\mathrm{D}$-waves had permanent motor deficits after surgery at long-term follow-up.

Furthermore, during our experience, the use of D-waves was also important for 2 additional reasons. 1) Often during surgery, the muscle MEP response is recorded in an on-off fashion; however, D-wave deterioration usually occurs gradually so that there is time to take corrective measures. ${ }^{17}$ 2) The D-wave allows a continuous monitoring of the function of the corticospinal tract without the need to interrupt the surgery for elicitation of the muscle MEPs; this real-time continuous monitoring was important mostly during dissection of tumors with a less well-defined border between lesion and healthy spinal cord tissue or in IDEM tumors located in an anterolateral position where rotation of the spinal cord can be monitored.

Finally, even if previous papers, such as that of Costa et al., ${ }^{3}$ reported that $\mathrm{D}$-wave monitoring provides relevant data as to motor outcome in a heterogeneous series of spinal pathologies (e.g., intramedullary tumors, intradural extramedullary tumors, cervical degenerative disorders), our study is the first to show the statistically proven superiority of D-wave monitoring in comparison with MEP and SSEP monitoring in terms of predictive value in a specific cohort of patients.

\section{Factors Correlated With IONM Changes}

The only independent risk factors associated with significant IONM changes in the entire population of this study were the location anterolateral to the spinal cord $(\mathrm{p}$ $=0.0001)$ and age greater than 65 years $(p=0.0001)$. In the literature, some studies have reported that a ventral location of IDEM tumors is associated with worse neurological outcome. Slin'ko and Al-Qashqish ${ }^{22}$ have shown that the more ventral the tumor was to the spinal cord, the more postoperative neurological deficits that occurred. Conversely, Ahn and colleagues ${ }^{1}$ found no association between ventral tumor location and unfavorable outcomes. Thus, the prognostic value of a ventral tumor location remains under debate. However, the surgical management of anterolateral IDEM tumors often requires spinal cord manipulation, and the use of IONM can help to prevent or mitigate irreversible neurological deficits.

\section{Limitations of the Study}

The limitations of our study include its retrospective nature, lack of randomization, and no comparison group of patients who underwent tumor removal without IONM. However, monitoring was performed by the same team throughout the study, and all operations were performed by senior surgeons (R.G. and F.S.), thus limiting a possible source of bias.

\section{Conclusions}

$\mathrm{D}$-wave monitoring was feasible in all patients without severe preoperative motor deficits. The use of D-wave monitoring demonstrated a statistically significant greater ability to predict postoperative deficits compared with SSEP and MEP monitoring alone and allowed us to proceed with IDEM tumor resection, even in cases of SSEP or MEP loss. Patients older than 65 years and with anterolateral IDEM tumors can benefit most from the use of IONM. For these reasons, in our series, the recording of D-waves significantly increased the accuracy and clinical value of neurophysiological monitoring in IDEM tumor resection.

\section{Acknowledgments}

We are grateful to Dr. Gabriella Torre, Dr. Francesca Ferrari, and Dr. Claudio Basile, Neurophysiology Unit, Neuromotor Department, IRCCS “Arcispedale Santa Maria Nuova” of Reggio Emilia, for technical help in monitoring the patients and for helping with the collection of data.

\section{References}

1. Ahn DK, Park HS, Choi DJ, Kim KS, Kim TW, Park SY: The surgical treatment for spinal intradural extramedullary tumors. Clin Orthop Surg 1:165-172, 2009

2. Choi I, Hyun SJ, Kang JK, Rhim SC: Combined muscle mo- 
tor and somatosensory evoked potentials for intramedullary spinal cord tumour surgery. Yonsei Med J 55:1063-1071, 2014

3. Costa P, Peretta P, Faccani G: Relevance of intraoperative D wave in spine and spinal cord surgeries. Eur Spine $\mathbf{J}$ 22:840-848, 2013

4. Deletis V, Kothbauer K: Intraoperative neurophysiology of the corticospinaltract, in Stålberg E, Sharma HS, Olsson Y (eds): Spinal Cord Monitoring. Vienna: Springer, 1998, pp 421-444

5. Deletis V, Sala F: Intraoperative neurophysiological monitoring of the spinal cord during spinal cord and spine surgery: a review focus on the corticospinal tracts. Clin Neurophysiol 119:248-264, 2008

6. Fehlings MG, Brodke DS, Norvell DC, Dettori JR: The evidence for intraoperative neurophysiological monitoring in spine surgery: does it make a difference? Spine (Phila Pa 1976) 35 (9 Suppl):S37-S46, 2010

7. Ghadirpour R, Nasi D, Iaccarino C, Giraldi D, Sabadini R, Motti L, et al: Intraoperative neurophysiological monitoring for intradural extramedullary tumors: why not? Clin Neurol Neurosurg 130:140-149, 2015

8. Hadley MN, Shank CD, Rozzelle CJ, Walters BC: Guidelines for the use of electrophysiological monitoring for surgery of the human spinal column and spinal cord. Neurosurgery 81:713-732, 2017

9. Harel R, Schleifer D, Appel S, Attia M, Cohen ZR, Knoller N: Spinal intradural extramedullary tumors: the value of intraoperative neurophysiologic monitoring on surgical outcome. Neurosurg Rev 40:613-619, 2017

10. Helseth A, Mørk SJ: Primary intraspinal neoplasms in Norway, 1955 to 1986 . A population-based survey of 467 patients. J Neurosurg 71:842-845, 1989

11. Jallo GI, Freed D, Epstein F: Intramedullary spinal cord tumors in children. Childs Nerv Syst 19:641-649, 2003

12. Joaquim AF, Almeida JP, Dos Santos MJ, Ghizoni E, de Oliveira E, Tedeschi H: Surgical management of intradural extramedullary tumors located anteriorly to the spinal cord. J Clin Neurosci 19:1150-1153, 2012

13. Korn A, Halevi D, Lidar Z, Biron T, Ekstein P, Constantini S Intraoperative neurophysiological monitoring during resection of intradural extramedullary spinal cord tumors: experience with 100 cases. Acta Neurochir (Wien) 157:819-830, 2015

14. Kothbauer KF, Deletis V, Epstein FJ: Motor-evoked potential monitoring for intramedullary spinal cord tumor surgery: correlation of clinical and neurophysiological data in a series of 100 consecutive procedures. Neurosurg Focus 4(5):e1, 1998

15. Lakomkin N, Mistry AM, Zuckerman SL, Ladner T, Kothari P, Lee NJ, et al: Utility of intraoperative monitoring in the resection of spinal cord tumors: an analysis by tumor location and anatomical region. Spine (Phila Pa 1976) 43:287-294, 2018

16. Malhotra NR, Shaffrey CI: Intraoperative electrophysiological monitoring in spine surgery. Spine (Phila Pa 1976) 35:2167-2179, 2010
17. Nasi D, Ghadirpour R, Servadei F: Intraoperative neurophysiologic monitoring in spinal intradural extramedullary tumors: only a prognostic tool? Neurosurg Rev 40:583-585, 2017

18. Sala F, Bricolo A, Faccioli F, Lanteri P, Gerosa M: Surgery for intramedullary spinal cord tumors: the role of intraoperative (neurophysiological) monitoring. Eur Spine J 16 (Suppl 2):S130-S139, 2007

19. Sala F, Palandri G, Basso E, Lanteri P, Deletis V, Faccioli F, et al: Motor evoked potential monitoring improves outcome after surgery for intramedullary spinal cord tumors: a historical control study. Neurosurgery 58:1129-1143, 2006

20. Sandalcioglu IE, Hunold A, Müller O, Bassiouni H, Stolke D, Asgari S: Spinal meningiomas: critical review of 131 surgically treated patients. Eur Spine J 17:1035-1041, 2008

21. Scibilia A, Terranova C, Rizzo V, Raffa G, Morelli A, Esposito $\mathrm{F}$, et al: Intraoperative neurophysiological mapping and monitoring in spinal tumor surgery: sirens or indispensable tools? Neurosurg Focus 41(2):E18, 2016

22. Slin'ko EI, Al-Qashqish II: Intradural ventral and ventrolateral tumors of the spinal cord: surgical treatment and results. Neurosurg Focus 17(1):ECP2, 2004

23. Sutter M, Eggspuehler A, Grob D, Jeszenszky D, Benini A, Porchet F, et al: The validity of multimodal intraoperative monitoring (MIOM) in surgery of 109 spine and spinal cord tumors. Eur Spine J 16 (Suppl 2):S197-S208, 2007

24. Van Goethem JWM, van den Hauwe L, Özsarlak O, De Schepper AM, Parizel PM: Spinal tumors. Eur J Radiol 50:159-176, 2004

25. Westwick HJ, Yuh SJ, Shamji MF: Complication avoidance in the resection of spinal meningiomas. World Neurosurg 83:627-634, 2015

\section{Disclosures}

Dr. Iaccarino: consultant for Finceramica S.p.A.

\section{Author Contributions}

Conception and design: Ghadirpour, Motti, Valzania, Servadei. Acquisition of data: Iaccarino, Romano, Motti. Analysis and interpretation of data: Nasi, Iaccarino, Sabadini, Servadei. Drafting the article: Nasi, Servadei. Critically revising the article: Ghadirpour, Romano, Motti, Servadei. Reviewed submitted version of manuscript: Ghadirpour, Sabadini, Valzania, Servadei. Approved the final version of the manuscript on behalf of all authors: Nasi. Statistical analysis: Nasi, Servadei. Administrative/technical/material support: Sabadini. Study supervision: Ghadirpour, Romano, Valzania, Servadei.

\section{Correspondence}

Davide Nasi: Umberto I General Hospital, Università Politecnica delle Marche, Ancona, Italy. davidenasi83@gmail.com. 